\title{
A limitation on Long's model in stratified fluid flows
}

\author{
By HAR VEY SEGUR \\ California Institute of Technology, Pasadena, California 91109
}

(Received 15 July 1970 and in revised form 21 December 1970)

The flow of a continuously stratified fluid into a contraction is examined, under the assumptions that the dynamic pressure and the density gradient are constant upstream (Long's model). It is shown that a solution to the equations exists if and only if the strength of the contraction does not exceed a certain critical value which depends on the internal Froude number. For the flow of a stratified fluid over a finite barrier in a channel, it is further shown that, if the barrier height exceeds this same critical value, lee-wave amplitudes increase without bound as the length of the barrier increases. The breakdown of the model, as implied by these arbitrarily large amplitudes, is discussed. The criterion is compared with available experimental results for both geometries.

\section{Introduction}

A basic problem in geophysical fluid mechanics is the flow of a density-stratified fluid over an obstacle. One of the most confusing aspects of this problem has been the question of the blocking of the flow by the obstacle, i.e. whether a given obstacle must fundamentally alter the flow far upstream of itself. The same question occurs in problems in rotating flows and water waves. Blocking, when it exists, invalidates those methods of analysis which prescribe the flow far upstream without regard to the obstacle. There is considerable interest, therefore, in determining the conditions under which blocking occurs.

Trustrum (1964) performed a linearized analysis for the transient flow created by a variety of small disturbances, both in stratified and in rotating flows. For a disturbance that was effectively a weak contraction of a rotating flow, she concluded that the upstream conditions depend on the means of setting up the steady motion as well as on the geometry of the problem. Benjamin (1970) carried a small perturbation analysis to the second order, and claimed that some upstream influence always accompanies lee-waves. He did not determine the relative importance of this upstream influence, or whether its omission seriously alters the solution. $\dagger$

Using the other useful analytical tool for continuous stratifications, Long $(1953 a, 1955)$ cast the steady equations of motion of an inviscid, incompressible fluid in a certain form (which is essentially Crocco's relationship) without regard to the size of the obstacle. Then he discovered particular boundary conditions

$\dagger$ Note added in proof. The editor has notified the author of recent work (in press) by Dr Michael E. McIntyre, which apparently does answer these questions. Under conditions for which some (perhaps negligible) upstream influence is necessary, Long's model must be considered an approximation, and the results of the present paper interpreted accordingly. 
upstream for which the equations reduce to the Helmholtz equation with inhomogeneous (but linear) boundary conditions. The linearity of the equations does not depend on the obstacle's being small, so that flow around physically important obstacles of finite size can be considered. In addition, Long's model possesses a certain generality. It has been shown that the equations also describe the flow around a symmetrical obstacle moving along the axis of a rotating fluid (Long $1953 b)$ and the meanderings of the zonal winds under the $\beta$-plane approximation (Ball 1959). Thus, the results which will be obtained here for stratified fluids can be extended to cover these other areas as well.

Unfortunately, the range of application of Long's model is unknown. The results of Trustrum and of Benjamin indicate that the boundary conditions upstream of an obstacle may depend on the obstacle itself. Thus, for a given obstacle, to specify particular boundary conditions upstream may be to overspecify the problem, and no solution may exist, or the solution may not be physically realizable.

In this paper a criterion is obtained which delimits the range of application of Long's model. Violation of the criterion implies that at least one of the assumptions of Long's model fails. When the model fails, the flow in question may not be steady, viscous effects may influence the whole flow field, or the nature of the flow postulated upstream may not be possible. Failure of Long's model is not implied by the presence of some (perhaps negligible) upstream influence of the obstacle, so this criterion is distinct from that obtained by Benjamin (1970).

In $\S 2$ we examine the steady two-dimensional flow of an inviscid stratified fluid into an arbitrary contraction. The method of constructing solutions developed by Drazin \& Moore (1967) yields a simple criterion (which involves the internal Froude number and the strength of the contraction) beyond which the whole model breaks down. The problem is analyzed in detail for abrupt contractions, and it is shown that the criterion is both necessary and sufficient for the existence of solutions to Long's model.

The flow of a stratified fluid past an obstacle of finite length is examined in $\S 3$. The key to the problem lies in the same construction technique. Solutions apparently exist for almost every obstacle of finite length. However, if the same criterion (which now depends on the obstacle height, rather than the contraction strength) is violated, the resulting flow pattern is so violent that the validity of the model is doubtful. Approximate lee-wave amplitudes for the flow past rectangular obstacles are obtained and discussed.

In $\$ 4$ this criterion is compared with available experimental results for both geometries. Whenever the criterion is violated in these experiments, the flow which results is found to be blocked in the sense that stagnant regions upstream of the obstacle exert a radical influence on the nature of the entire flow field.

\section{Flow into contractions}

\subsection{General derivation}

We consider first the steady two-dimensional flow of an inviscid incompressible stratified fluid into an arbitrary contraction. The equations of motion can be 
reduced to a single non-linear partial differential equation for the stream function, $\psi$, of the form (Dubreil-Jacotin 1935)

$$
\rho \nabla^{2} \psi+\frac{d \rho}{d \psi}\left\{\frac{1}{2}|\nabla \psi|^{2}+g y\right\}=\frac{d H}{d \psi},
$$

where $\rho$ is the density, $p$ is the pressure and

$$
H(\psi)=p+\rho g y+\frac{1}{2} \rho|\nabla \psi|^{2} .
$$

One makes all lengths dimensionless using the characteristic length $H / \pi$, where $H$ is the channel depth, and defines $y_{0}(x, y)$ to be the dimensionless elevation that

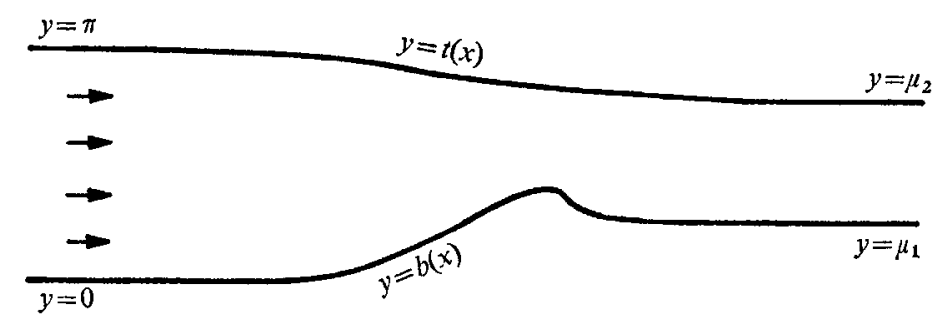

FIgURE 1. Typical geometry for the flow into a contraction.

the streamline passing through the point $(x, y)$ attains far upstream. Long $(1953 a)$ showed that if the flow upstream satisfies the two conditions

and

$$
\begin{aligned}
& \left.\rho\left(y_{0}\right) U^{2}\left(y_{0}\right)=\text { const. }>0\right) \\
& \left.d \rho / d y_{0}=\text { const. }<0\right\} \\
& \text { then }(2.1) \text { becomes } \quad \nabla^{2} \delta+k^{2} \delta=0 \text {, } \\
& \delta(x, y)=y_{0}(x, y)-y \\
& k^{2}=-\frac{g\left(d \rho / d y_{0}\right) H}{\rho U^{2} \pi} .
\end{aligned}
$$$$
\text { where }
$$

and

The boundary conditions for $\delta$ are (see figure 1)

$$
\begin{aligned}
& \delta \rightarrow 0 \text { as } x \rightarrow-\infty \\
& \delta \text { bounded as } x \rightarrow+\infty \\
& \delta=-b(x) \text { along the bottom wall, } y=b(x) ; \\
& \delta=\pi-t(x) \text { along the top wall, } y=t(x) ;
\end{aligned}
$$

where

$$
\begin{aligned}
& b(x) \rightarrow 0, \quad t(x) \rightarrow \pi \quad \text { as } \quad x \rightarrow-\infty, \\
& b(x) \rightarrow \mu_{1}, \quad t(x) \rightarrow \mu_{2} \quad \text { as } \quad x \rightarrow+\infty .
\end{aligned}
$$

To assure uniqueness, it is necessary to exclude values of $k$ for which either $k=m$ or $k\left(\mu_{2}-\mu_{1}\right) / \pi=m$ for any integer $m$. These values denote resonant flow situations, in which one would expect the assumptions of the model to fail.

The assumptions (2.2) reduce the non-linear equations of motion to a linear boundary-value problem, regardless of the obstacle size. Drazin \& Moore (1967) further noted that, since the governing equation is the Helmholtz equation, one acquires the use of the powerful tools of diffraction theory. In particular, the 
stratified flow problem can be replaced by an equivalent acoustic problem. Thus, one considers the superposition of the effects of $(a)$ a set of sources, distributed continuously along the walls of the contraction, and $(b)$ a source at $x=+\infty$. The sources in the contraction continuously emit waves that travel both upstream and downstream, and one must choose the source at $x=+\infty$ in such a way as to annihilate any disturbance upstream. The upstream disturbance from the sources in the contraction can be obtained simply by separating variables in (2.3). Solutions to (2.3) are $X(x) \sin n y$, where $X(x)$ satisfies

$$
X^{\prime \prime}(x)+\left(k^{2}-n^{2}\right) X(x)=0 .
$$

Thus, undamped waves can exist upstream for $k>1$. The composite of all the waves which travel upstream of the contraction is of the form

$$
\delta=\sum_{n=1}^{[k]} A_{n} \exp \left[i\left(k^{2}-n^{2}\right)^{\frac{1}{2}} x\right] \sin n y+\sum_{n=[k]+1}^{\infty} A_{n} \exp \left[\left(n^{2}-k^{2}\right)^{\frac{1}{2}} x\right] \sin n y,
$$

where $[k]$ denotes the greatest integer less than or equal to $k$.

If the contraction is symmetric about the line $y=\frac{1}{2} \pi$ (so that $b(x)=\pi-t(x)$ in figure 1), the solution also must be symmetric, and no odd wave modes enter into the solution. For this exceptional geometry, all the statements of this section hold if one replaces $(k)$ by $\left(\frac{1}{2} k\right)$. Thus, for a symmetric contraction, undamped waves exist upstream for $k>2$.

We now add to this problem the other wave generator at $x=+\infty$, whose waves travel upstream. This additional wave generator is to be adjusted to send sinusoidal waves to $x=-\infty$ which exactly cancel the existing waves

$$
\sum_{n=1}^{[k]} A_{n} \exp \left[i\left(k^{2}-n^{2}\right)^{\frac{1}{2}} x\right] \sin n y
$$

so that the boundary condition upstream is satisfied. Then the sum of all the waves emitted by the walls, plus those generated at $x=+\infty$, satisfies the boundary-value problem (2.3). Drazin \& Moore (1967) used this equivalent formulation to construct solutions for the flow over obstacles of finite length. Grimshaw (1968) later proved the uniqueness of these solutions, provided only that the obstacle satisfies a certain convexity condition and has finite length.

Let us apply this equivalent formulation of the boundary-value problem (2.3) to the contraction shown in figure 1 . We define $w$, the asymptotic contraction ratio, by

$$
w=\left(\mu_{2}-\mu_{1}\right) / \pi
$$

We now show that the model breaks down irreparably if $w$ is too small. The wave generator at $x=+\infty$ can only emit sinusoidal waves of the form

$$
\sum_{n=1}^{[k w]} B_{n} \exp \left[i\left(k^{2}-(n / w)^{2}\right)^{\frac{1}{2}} x\right] \sin \left\{(n / w)\left(y-\mu_{1}\right)\right\}
$$

In this construction technique, the amplitudes, $B_{n}$, of these [ $\left.k w\right]$ wave modes are to be adjusted to cancel the $[k]$ existing wave modes upstream. If $[k] \geqslant 1$, however, there exists a range of contraction ratios such that $[k]>[k w]$. Obviously, 
no set of $[k w]$ wave amplitudes can be determined which cancel $[k]$ existing waves if $[k]>[k w]$. Thus,

$$
[k]=[k w]
$$

emerges as a requirement for the existence of solutions to the boundary-value problem (2.3) for any asymmetric contraction, regardless of its local shape. Similarly,

$$
\left[\frac{1}{2} k\right]=\left[\frac{1}{2} k w\right]
$$

must be satisfied for any symmetric contraction if Long's model is to describe the flow into that contraction.

\subsection{Abrupt contractions}

We can make these arguments precise if we restrict our attention to abrupt contractions, of the type shown in figure 2. The advantage of this simple geometry is that separation of variables yields the form of the solution for $x<0$ and

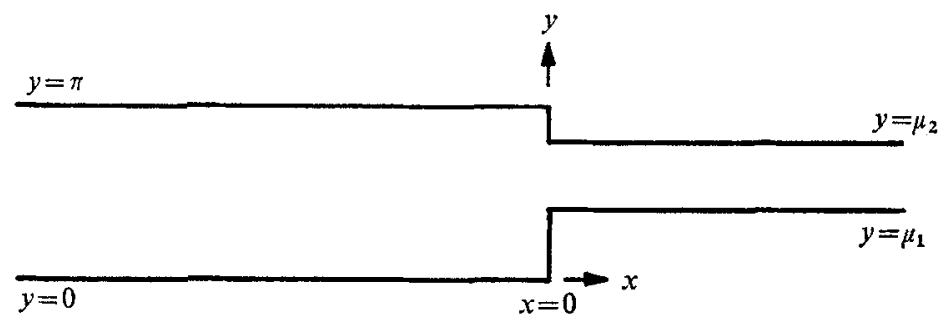

Figure 2. An abrupt contraction.

for $x>0$, which can then be matched at $x=0$ to obtain the solution to the boundary-value problem (2.3). Thus, for $x<0$,

$$
\delta=\sum_{n=[k]+1}^{\infty} \frac{A_{n}}{n^{\frac{1}{2}}} \exp \left[\left(n^{2}-k^{2}\right)^{\frac{1}{2}} x\right] \sin n y,
$$

where $\left\{A_{n}\right\}$ is a sequence of real, undetermined constants, and

One defines $\gamma$ and $\tilde{y}$ by

$$
\sum_{n=[k]+1}^{\infty} A_{n}^{2} / n<\infty . \dagger
$$

$$
-\mu_{1}=\gamma \sin k\left(\mu_{1}-\tilde{y}\right), \quad \pi-\mu_{2}=\gamma \sin k\left(\mu_{2}-\tilde{y}\right) .
$$

Then, for $x>0$,

$$
\begin{aligned}
\delta(x, y) & =\sum_{r=1}^{[k w]}\left[\frac{b_{r}}{r^{\frac{1}{2}}} \sin \left\{\left(k^{2}-\left(\frac{r}{w}\right)^{2}\right)^{\frac{1}{2}} x\right\}+\frac{a_{r}}{r^{\frac{1}{2}}} \cos \left\{\left(k^{2}-\left(\frac{r}{w}\right)^{2}\right)^{\frac{1}{2}} x\right\}\right] \sin \left\{\frac{r}{w}\left(y-\mu_{1}\right)\right\} \\
& +\sum_{r=[k w]+1}^{\infty} \frac{a_{r}}{r^{\frac{1}{2}}} \exp \left[-\left(\left(\frac{r}{w}\right)^{2}-k^{2}\right)^{\frac{1}{2}} x\right] \sin \left\{\frac{r}{w}\left(y-\mu_{1}\right)\right\}+\gamma \sin k(y-\tilde{y}) .
\end{aligned}
$$

$\dagger$ The normalization of the Fourier coefficients shown here was suggested by Professor H. B. Keller. It makes the infinite matrix that will appear in (2.7) symmetric and is crucial for this method of proof. 
$\delta(x, y)$ and $\partial \delta(x, y) / \partial x$ must be continuous at $x=0$. Carrying out this matehing, and eliminating $\left\{b_{r}\right\}_{1}^{[k w\}},\left\{a_{r}\right\}_{\mid k w\}+1}^{\infty}$, one obtains the following infinite set of linear algebraic equations for $\left\{a_{n}\right\}_{1}^{[k w]}$ and $\left\{A_{n}\right\}_{[k]+1}^{\infty}$ :

$$
\begin{aligned}
w \sum_{n=1}^{[k w]} \frac{(n m)^{\frac{1}{2}}}{n^{2}-(m w)^{2}}\left\{\sin m \mu_{1}+(-1)^{n+1} \sin m \mu_{2}\right\} a_{n} \\
\quad-\frac{2}{\pi}(w)^{2} \sum_{\substack{n=[k \mid+1 \\
n \neq m}}^{\infty} \frac{(m n)^{\frac{1}{2}}\left(1-(k / n)^{2}\right)^{\frac{1}{2}}}{m^{2}-n^{2}}\left\{\left[\sin m \mu_{1} \sin n \mu_{1}+\sin m \mu_{2} \sin n \mu_{2}\right]\right. \\
\left.\times[W(m)-W(n)]-\left[\sin m \mu_{1} \sin n \mu_{2}+\sin m \mu_{2} \sin n \mu_{1}\right][X(m)-X(n)]\right\} A_{n} \\
\quad-\left(\frac{\pi}{2}+\frac{2}{\pi}(w)^{2}\left(m^{2}-k^{2}\right)^{\frac{1}{2}}\left\{\left[\sin ^{2} m \mu_{1}+\sin ^{2} m \mu_{2}\right] Y(m)\right.\right. \\
\left.\left.\quad-2 \sin m \mu_{1} \sin m \mu_{2} Z(m)\right\}\right) A_{m} \\
=-\frac{k^{2}}{m^{\frac{1}{2}}\left(m^{2}-k^{2}\right)}\left\{\mu_{1} \cos m \mu_{1}+\left(\pi-\mu_{2}\right) \cos m \mu_{2}\right\}-\frac{1}{m^{\frac{3}{2}}}\left\{\sin m \mu_{2}-\sin m \mu_{1}\right\} \\
\quad+\frac{k m^{\frac{1}{2}}}{\left(m^{2}-k^{2}\right) \sin k w \pi}\left\{\left(\pi-\mu_{2}\right)\left(\sin m \mu_{1}-\cos k w \pi \sin m \mu_{2}\right)\right. \\
\left.\quad-\mu_{1}\left(\sin m \mu_{2}-\sin m \mu_{1} \cos k w \pi\right)\right\}
\end{aligned}
$$

where

$$
\begin{gathered}
d(m, r)=\left(r^{2}-(m w)^{2}\right)^{-1}, \quad s(r)=\left(r^{2}-(k w)^{2}\right)^{-\frac{1}{2}}, \\
W(m)=m^{2} \sum_{r=[k w]+1}^{\infty} d(m, r) s(r), \\
X(m)=m^{2} \sum_{r=[k w]+1}^{\infty}(-1)^{r} d(m, r) s(r), \\
Y(m)=\sum_{r=[k w]+1}^{\infty} r^{2} d^{2}(m, r) s(r), \\
Z(m)=\sum_{r=[k w]+1}^{\infty}(-1)^{r} r^{2} d^{2}(m, r) s(r) .
\end{gathered}
$$

The assumptions (2.2) reduced the non-linear equations of motion to the Helmholz equation with inhomogeneous boundary conditions (2.3). This linear differential equation has now been replaced by an infinite set of linear algebraic equations (2.7). If (2.7) has a solution for which

$$
\sum_{n=[k]+1}^{\infty} \frac{A_{n}^{2}}{n}<\infty
$$

then (2.3) has a bounded solution, and conversely.

Before examining the characteristics of (2.7) we note one important specialization of it. In the case of a symmetric contraction, in which

$$
\mu_{1}=\pi-\mu_{2}
$$

one notes that the right-hand side of every equation with odd $(m)$ in $(2.7)$ vanishes. Furthermore, every element of the matrix on the left-hand side for which $(m+n)$ 
is odd vanishes. It follows that (2.7) can be separated into two sets of linear algebraic equations, a homogeneous set for the odd Fourier coefficients, $\left\{a_{2 n-1}\right\}$ and $\left\{A_{2 n-1}\right\}$, and an inhomogeneous set for the even coefficients, $\left\{a_{2 n}\right\}$ and $\left\{A_{2 n}\right\}$. Hence, if the matrices for each set have well-defined inverses, then the odd coefficients must all vanish, so that the solution to (2.3) is described completely by the even coefficients. Thus, the flow itself must also be symmetric, as mentioned in $\$ 2.1$.

The set of equations (2.7) can be written in the following matrix form:

$$
E . v=f
$$

where $f=\left\{f_{m}\right\}_{m=1}^{\infty}$ denotes an infinite sequence, the $m$ th element of which is the right-hand side of the $m$ th equation of (2.7), and $v$ is an infinite sequence with

$$
v_{n}=\left\{\begin{array}{l}
a_{n} \text { for } \quad 1 \leqslant n \leqslant[k w], \\
A_{n+([k]-[k w)} \text { for } n>[k w],
\end{array}\right.
$$

and $E=\left(\left(E_{m n}\right)\right)$, in which $E_{m n}$ is the coefficient of $v_{n}$ in the $m$ th equation of (2.7).

The behaviour of $f$ and $E$ as functions of $(m, n)$ is needed to determine the existence of solutions to (2.7). For any given combination of $\left(k, \mu_{1}, \mu_{2}\right)$, as $m \rightarrow \infty$,

$$
f_{m}=O\left(m^{-\frac{3}{2}}\right) \text {. }
$$

We refer to the elements of the matrix in (2.7) which involve $Y(m)$ and $Z(m)$ as 'dominant elements'. For any $n<[k w]$, we also eall the elements for which $|n-m w|$ is a minimum a 'dominant element'. One can show that each of these elements is bounded. If $e(m, m)$ denotes the dominant element in the $m$ th row, then for $m>[k]+1$,

$$
-\infty<e(m, m)<-\frac{1}{2} \pi \text {. }
$$

All the other elements of the matrix are called 'non-dominant elements', and are denoted by $e(m, n)$. If $n<[k w]$, it follows that

If $n>[k w]$,

$$
e(m, n)=O\left(m^{-\frac{3}{2}}\right) \text { as } \quad m \rightarrow \infty .
$$

$$
e(m, n)=O\left(\frac{(n m)^{\frac{1}{2}}}{m^{2}-n^{2}} \ln \frac{m}{n}\right) \quad m \neq n, \quad \text { as } \quad n \rightarrow \infty \text { or as } \quad m \rightarrow \infty .
$$

Thus, as $(m+n)$ increases, every element in any row or column tends to zero, except those along one diagonal, where the elements remain finite. The significance of the criterion (2.5) is that this dominant diagonal become the centre diagonal of the matrix for $[k]=[k w]$. It is shown in appendix A that this criterion is both necessary and sufficient for the existence of a bounded inverse to the matrix in (2.7). That result may be stated as follows:

For an asymmetric, abrupt contraction, a bounded solution to (2.3) exists uniquely if

$$
[k]=[k w] .
$$

If $[k]>[k w]$, no bounded solution to $(2.3)$ exists unless the $([k w]+1)$ th, $\ldots$, $([k])$ th equations of (2.7) have vanishing right-hand sides. 
For a symmetric, abrupt contraction, a bounded solution to (2.3) exists uniquely if

$$
\left[\frac{1}{2} k\right]=\left[\frac{1}{2} k w\right] \text {. }
$$

If $\left[\frac{1}{2} k\right]>\left[\frac{1}{2} k w\right]$, no bounded solution to $(2.3)$ exists unless the $\left(\left[\frac{1}{2} k w\right]+1\right)$ th, ... ([ $\left.\left.\frac{1}{2} k\right]\right)$ th equations corresponding to $(2.7)$ for symmetric contractions have vanishing right-hand sides. The reader is referred to the appendix for details.

The range of application of Long's model for contractions can thus be explicitly defined. The model is inappropriate unless (2.5) is satisfied. This is confirmed experimentally in $\S 4$.

\section{Flow past obstacles}

The strength of the criterion (2.5) suggests that it might have some counterpart for the flow over obstacles of finite length. If one seeks an existence criterion corresponding to (2.5), then it apparently has no counterpart. In terms of the construction technique used in $\$ 2.1$, such an existence criterion would mean that, within some range of the parameters, an obstacle of finite length could reflect completely a wave emitted from the wave generator at $x=+\infty$. Drazin \& Moore (1967) conjectured that any finite obstacle that allowed some gap above it must transmit some energy from the wave emitted at $x=+\infty$. Grimshaw (1968) commented that, since the problem is linear, a proof of uniqueness suggests strongly that existence can also be demonstrated.

But, if solutions always exist, how could a criterion like (2.5) affect the problem? It is not difficult to show that the criterion must significantly affect the transmission coefficient of the obstacle, and that it must become more important for longer obstacles. In the terminology of $\$ 2.1,[k]$ sinusoidal wave modes are generated downstream, which must be transmitted past the obstacle. If $[k]>[k w]$, where $w$ now denotes the ratio of the fluid depth over the obstacle to that upstream, an insufficient number of sinusoidal wave modes exist over the obstacle to carry all the wave energy generated at $x=+\infty$. It follows that part of this energy must be transmitted by the exponential modes (see figure 3 )

$$
\sum_{n=[k w]+1}^{\infty} D_{n} \exp \left[\left(\left(\frac{n}{w}\right)^{2}-k^{2}\right)^{\frac{1}{2}}(x-L)\right] \sin \left\{\frac{n}{w}(y-h)\right\} .
$$

If the obstacle is long, only a small fraction of the incident energy can be transmitted by these exponential modes. In order to cancel the waves at $x=-\infty$, therefore, the energy input at $x=+\infty$ must be tremendous. Hence, if $[k]>[k w]$ and the obstacle is long, a violent wave pattern in the lee of the obstacle is required if no waves are to exist upstream.

As in $\S 2$, the argument can be made more precise by examining a particular class of obstacles. The flow past long rectangular barriers, like the one shown in figure 3 , can be analyzed by the same matching techniques used above. The algebra is twice as long, and is relegated to appendix B. Instead of the infinite matrix obtained in (2.7), one obtains a doubly infinite matrix $\left(E_{n n}\right)$, where $-\infty<m, n<\infty$. The importance of the criterion (2.5) is that this matrix is well behaved if $[k]=[k w]$, but becomes more nearly singular as $L$ increases if 
$[k]>[k w]$. If $\left\{C_{n} / n^{\frac{1}{2}}\right\}_{n=1}^{[k]}$ denote the amplitudes of the different lee-wave modes, the following approximate result is obtained in appendix B:

$$
\begin{gathered}
\text { if } \quad[k]=[k w] \quad C_{n}=O(1) \text { as } L \rightarrow \infty ; \\
\text { if } \quad[k]>[k w] \quad \operatorname{ord}\left(C_{n}\right)>L \text { as } L \rightarrow \infty,
\end{gathered}
$$

i.e.

$$
C_{n} / L \rightarrow \infty \text { as } L \rightarrow \infty \text {. }
$$

Thus, if (2.5) is violated for long obstacles (large $L$ ), the solution to Long's model contains a violent pattern of lee-waves whose amplitudes grow without bound as $L$ increases. In light of such an unrealistic result, one must question the validity

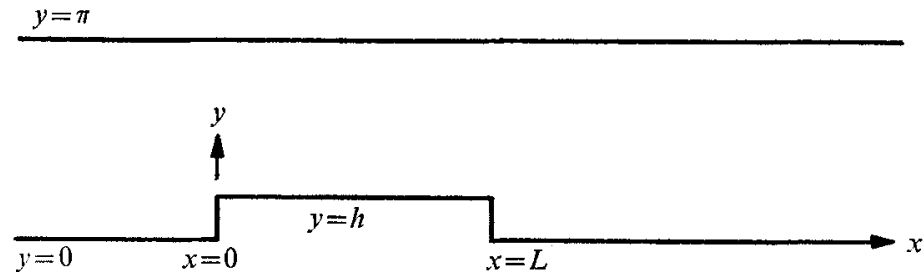

Figure 3. Rectangular obstacle in a channel.

of the assumptions of the model. A comprehensive critique of these assumptions is beyond the scope of this paper. We examine briefly the assumption of an inviscid fluid, whose breakdown can be verified directly from (3.1).

An inviscid model neglects any effects of viscous forces in determining the flow, an assumption whose validity can be tested $a$ posteriori by determining whether the inviscid solution approximately satisfies the viscous equations in the domain. When the inviscid solution contains violent wave-patterns, as it does for large $L$ when (2.5) is violated, the validity of this assumption is doubtful. In fact, one can use (3.1) to estimate the obstacle length required to invalidate the inviscid approximation. With constant viscosity, $\mu$, the equations of motion yield an energy equation

$$
(D / D t)\left\{p+\rho g y+\frac{1}{2} \rho|\nabla \psi|^{2}\right\}=\mu \nabla \psi \cdot \nabla\left(\nabla^{2} \psi\right)
$$

and a vorticity equation, corresponding to (2.1),

$$
\frac{D}{D t}\left\{\rho \nabla^{2} \psi+\frac{d \rho}{d \psi}\left(|\nabla \psi|^{2}+y g\right)\right\}=\mu \nabla^{2} \nabla^{2} \psi
$$

where

$$
(D / D t)\{\}=\mathbf{u} \cdot \nabla\{\} .
$$

Far enough downstream of the obstacle, the inviscid solution is given by

$$
y_{0}=y+\sum_{n=1}^{[k]} \frac{C_{n}}{n^{\frac{1}{2}}} \sin \left\{\left(k^{2}-n^{2}\right)^{\frac{1}{2}}\left(x-x_{n}\right)\right\} \sin n y
$$

where

$$
\psi=\int^{y_{0}} U(\eta) d \eta
$$

The $\left\{C_{n}\right\}$ depend on $L$, as shown in (3.1). One substitutes (3.3), with (3.1), into 
(3.2) to determine the minimum $L$ beyond which viscous effects in the lee of the barrier invalidate the use of an inviscid model. Using the definitions

$$
R e=\rho U H / \pi \mu, \quad \beta=-(1 / \rho) d \rho / d y_{0},
$$

one obtains the following results:

(a) If $[k]=[k w]$, wave amplitudes are bounded as $L \rightarrow \infty$. An inviscid analysis yields a valid approximation to the viscous solution for large $R e$, except in such singular regions as boundary layers and closed rotors.

(b) If $[k]>[k w]$, viscous effects are important everywhere downstream of the barrier unless either

or

$$
\text { (i) } \beta^{-1} L<1 \text { and } L=o\left(k^{-2} R e^{\frac{1}{2}}\right)
$$

(ii) $\beta^{-1} L \geqslant 1$ but $L=o\left(k^{-1} \beta^{-2} R e^{\frac{1}{4}}\right)$.

This establishes (2.5) as a necessary criterion for the validity of Long's inviscid model for the flow of a real fluid over long barriers, where 'long' is defined by (3.4). We note that this model might fail for other reasons (gravitational or shear instability, for example), and that some constraint other than (2.5) actually might be more stringent. Thus, the criterion (2.5) is not sufficient to ensure the validity of the model, but it is necessary.

\section{Comparison with experimental results}

\subsection{Contractions}

The only experimental results on stratified flow into a contraction with which the author is familiar were conducted by Debler (1959). In those experiments the contraction was a line sink in the corner of a channel of finite height, so that $w \doteq 0$. Fortunately, the contraction was also one-sided. Equation (2.10) states that a solution of (2.3) exists if and only if $k<1$. Experimentally, Debler found that the sink drew fluid from the whole channel for $k<1 \cdot 14(F>0.28$ in his notation, since his $F=1 / k \pi)$, and Yih (1965) showed that Long's model accurately described the flow. For $k>1 \cdot 14$, an essentially stagnant layer filled the upper part of Debler's channel, so that (2.2) was violated. Moreover, Debler's method of velocity measurement suggests that the effects of viscous boundary layers might account for the difference between the theoretical $(k<1)$ and the experimental $(k<1 \cdot 14)$ criteria. Thus, for the two-dimensional sink flow studied by Debler, the non-existence of solutions to Long's model implies a strong form of blocking.

\subsection{Barriers}

Laboratory experiments on the channel flow of a stratified fluid at relatively high Reynolds numbers over a barrier have been conducted by Long (1955) and by Davis (1969). Unfortunately, Davis used rather short, abrupt barriers. Using the definitions given in $\S 3$, he used barriers either with $L=2$ or with $L=0$, in a flow field with $R e$ ranging from 1300 to 5000 , and $\beta^{-1}$ ranging from 30 to 70 . Thus, his barriers were short enough to satisfy (3.4), so that the criterion (2.5) does not necessarily apply. It is worth mentioning that, with these abrupt barriers, Davis 
noticed that the downstream flow field was still not described by solutions to Long's model, because of large-scale separation behind the barrier crest.

Long conducted seven experiments (his figures $6-12$ ) for which $[k]=[k w]$. Computed solutions to the inviseid equations closely approximated the observed flow fields for these experiments. Although he observed blocked regions in some of the experiments, the blocking apparently was confined to a narrow layer ahead of the obstacle, with little effect outside that region. In two other experiments (his figures 13-14), for which he believed that no solution to Long's model existed, he demonstrated that a large enough barrier could create flow conditions upstream quite different from those described by (2.2). $[k]>[k w]$ for each of these experiments, although the barrier was long in the sense of (3.4) only for the second experiment.

Thus, the limited experimental evidence available is consistent with the result given above, that Long's model cannot describe the flow of a stratified fluid over a long barrier unless (2.5) is satisfied. In addition, it is consistent with the suggestion that Long's model might break down for reasons other than those implied by (2.5).

The author gratefully acknowledges the help he received from Drs Jorg Imberger, J. V. Wehausen, G.M. Corcos, F.S. Sherman, all of the University of California, Berkeley. The work on contractions was carried out while the author was a graduate student at Berkeley. He also thanks Drs H. B. Keller and G. B. Whitham of the California Institute of Technology for their helpful comments on the presentation of the material. Various parts of this work were supported by the National Aeronautics and Space Administration, the National Science Foundation and the Office of Naval Research, U.S. Navy.

\section{Appendix A. An existence criterion for contractions}

We seek to prove the existence criterion (2.10) in this appendix. We shall prove (2.10) for any contraction if $k<1$. If $k>1$, we shall prove (2.10) for any one-sided contraction or for any symmetric contraction.

\section{Existence}

If $(2.10 a)$ is satisfied, then $[k]=[k w]$ and all the dominant elements of the matrix in (2.7) lie along its main diagonal. We consider as a prototype the case $[k]=[k w]=0$. The body of the proof is identical for any other value of $[k](=[k w])$, and we shall indicate below the modifications necessary to generalize the proof.

A second restriction which greatly simplifies the proof is to consider only one-sided contractions. For definiteness, we take

$$
\mu=0, \pi-\mu_{2} \neq 0 .
$$

Then, corresponding to (2.9), the dominant elements of the matrix, which are also the diagonal elements, obey

where $0<\alpha(m)<4 / \pi^{2}$.

$$
-\infty<e(m, m)<-\left(\frac{1}{2} \pi+2 \pi w \alpha(m)\right)
$$


The non-dominant elements, which lie off the main diagonal, are given approximately by

$$
e(m, n) \doteq \frac{2}{\pi} \sin m \mu_{2} \sin n \mu_{2} \frac{(n m)^{\frac{1}{2}}}{m^{2}-n^{2}} \ln \frac{m}{n}
$$

It follows that the matrix in (2.7) can be written as

$$
E=-A+B
$$

where

$$
\begin{array}{rlrl}
A_{m n} & =-e(m, m) & \text { if } m=n, \\
& =0 & \text { if } m \neq n ; & \\
B_{m n} & \doteq \frac{2}{\pi} \sin m \mu_{2} \sin n \mu_{2} \frac{(n m)^{\frac{1}{2}}}{m^{2}-n^{2}} \ln \frac{m}{n} & \text { if } m \neq n, \\
& =0 & \text { if } m=n .
\end{array}
$$

Certainly $A^{-1}$ exists. In fact,

$$
\begin{aligned}
& \left(A^{-1}\right)_{m n}=-\frac{1}{e(m, m)} \quad \text { if } \quad m=n, \\
& =0 \quad \text { if } \quad m \neq n \text {. }
\end{aligned}
$$

Let $\left|B A^{-1}\right|$ denote the norm of the matrix $B A^{-1}$. Then $\left|B A^{-1}\right|<1$ implies that $E^{-1}$ exists and that $E^{-1}$ is given by the Neumann series: $\dagger$

$$
E^{-1}=-A^{-1}\left(I+B A^{-1}+B A^{-1} B A^{-1}+\ldots\right) .
$$

But

and, from (A 2),

$$
\left|B A^{-1}\right|<|B| \cdot\left|A^{-1}\right|
$$

$$
\left|A^{-1}\right|<2 / \pi \text {. }
$$

Therefore, if $|B| \leqslant \frac{1}{2} \pi$, then $E^{-1}$ exists. Now the norm of $B$ is given by

$$
|B|=\max \left\{\left|\sum_{\substack{m=1 \\ m \neq n}}^{\infty}\left(\sum_{n=1}^{\infty} \frac{2}{\pi} \sin m \mu_{2} \sin n \mu_{2} \frac{(n m)^{\frac{1}{2}}}{m^{2}-n^{2}} \ln \left(\frac{m}{n}\right) x_{n}\right) x_{m}\right| \sum_{m=1}^{\infty} x_{m} x_{m}\right\}
$$

for all $\left\{x_{m}\right\} \in l^{2}$. Certainly

$$
|B| \leqslant \max _{\left\{x_{m}\right\}}\left\{\left|\sum_{\substack{m=1 \\ m \neq n}}^{\infty}\left(\sum_{n=1}^{\infty} \frac{2}{\pi} \frac{(n m)^{\frac{1}{2}}}{m^{2}-n^{2}} \ln \left(\frac{m}{n}\right) x_{n}\right) x_{m}\right| \mid \sum_{m=1}^{\infty} x_{m} x_{m}\right\} .
$$

We refer to a theorem by Hardy, Littlewood \& Polya, in the form due to Schur: $\ddagger$

Suppose that $M(x, y)$ has the following properties:

(1) $M$ is non-negative, and homogeneous of degree -1 ;

(2) $\int_{0}^{\infty} M(x, 1) x^{-\frac{1}{2}} d x=\int_{0}^{\infty} M(1, y) y^{-\frac{1}{2}} d y=\gamma$;

(3) $M(x, 1) x^{-\frac{1}{2}}$ is a strictly decreasing function of $x$, and $M(1, y) y^{-\frac{1}{2}}$ of $y$.

Then

$$
\begin{aligned}
\sum_{m=1}^{\infty} \sum_{n=1}^{\infty} M(m, n) a_{m} b_{n} & <\gamma\left(\sum_{m=1}^{\infty} a_{m}^{2}\right)^{\frac{1}{2}}\left(\sum_{n=1}^{\infty} b_{n}^{2}\right)^{\frac{1}{2}} \text { unless }\left\{a_{n}\right\} \text { or }\left\{b_{n}\right\} \text { is null. } \\
& \dagger \text { For the proof seo Friedman (1956, p. 34). } \\
& \ddagger \text { Hardy, Littlewood \& Polya (1952, p. 227). }
\end{aligned}
$$


One verifies by inspection that

$$
B(x, y)=\frac{2}{\pi} \frac{(x y)^{\frac{1}{2}}}{x^{2}-y^{2}} \ln \frac{x}{y}
$$

satisfies the requirements of the theorem. Thus, $\gamma$ is defined by

$$
\gamma=\frac{2}{\pi} \int_{0}^{\infty} \frac{1}{x^{2}-1} \ln x d x
$$

The value of the definite integral is $\frac{1}{4} \pi^{2}$. The theorem (A 8) then states that

$$
|B|<\frac{1}{2} \pi,
$$

from which it follows that $E^{-1}$ exists. Therefore a solution to $(2.7)$ exists, it is unique and, from (2.8), it is within $l^{2}$. This proves the existence part of $(2.10 a)$ for one-sided contractions and for $[k]=0$.

The restriction $[k]=0$ can be removed with some juggling, the purpose of which is to put the matrix $E$ into a form such that the same proof applies for any finite $[k]$. If $[k]$ is finite, the convergence of $\left\{v_{n}\right\}$ is not affected if one redefines its first [k] elements such that

$$
v_{n}=a_{n} \frac{e(n, n)}{\left(-\frac{1}{2} \pi\right)} \quad(n \leqslant[k w])
$$

where $e(n, n)$ is the dominant element corresponding to $v_{n}$. One can then redefine the elements of the first $[k w]$ columns of $E$ in the following fashion:

$$
e(m, n)=\frac{(n m)^{\frac{1}{2}}}{n^{2}-(m w)^{2}}(w)\left\{(-1)^{n+1} \sin m \mu_{2}\right\} \frac{-\frac{1}{2} \pi}{e(n, n)} .
$$

Then, for all the dominant elements of $E$,

$$
e(m, m) \leqslant-\frac{1}{2} \pi \text {. }
$$

Furthermore, the sequence $e(m, n)$ in any column of $E$ for which $n \leqslant[k w]$ is majorized by some sequence that satisfies (A 3), so that (A 9) still holds. Thus $E^{-1}$ exists for any one-sided contraction.

One applies the same reasoning to the equations for a symmetric contraction to prove that, if $(2.10 b)$ is satisfied, then the matrices in the two sets of equations for the odd and even Fourier coefficients each have well-behaved inverses. Hence, all the odd wave modes vanish in a symmetric contraction, as advertised. Alternatively, if one replaces a symmetric contraction with the juxtaposition of two opposed one-sided contractions (replacing $k$ with $\frac{1}{2} k$ ), then the existence part of $(2.10 b)$ is established by inspection, giving $(2.10 a)$.

Further, one can juxtapose one-sided contractions to create any contraction, if $k<1$. The solution being composed entirely of exponential modes, it follows that the flow into any contraction, symmetric or not, must include one horizontal streamline. The flow on either side of this streamline may be regarded as the flow into a one-sided contraction (with $k^{2}$ properly adjusted), so that the existence part of (2.10) is established for any contraction if $k<1$.

To prove that (2.10) is a sufficient condition for the existence of solutions in any contraction is rather complicated. The upper bound (A 9) was quite close, and 
relied heavily on the fact that the symmetric matrix $B$ could be made nonnegative without increasing its norm too much. In the general case, in which non-dominant elements are given by

$$
\underset{m \neq n}{B_{m n}} \doteq\left\{\sin m \mu_{1} \sin n \mu_{1}+\sin m \mu_{2} \sin n \mu_{2}\right\} \frac{(m n)^{\frac{1}{2}}}{m^{2}-n^{2}} \ln \frac{m}{n},
$$

the same procedure does not yield a close enough bound to prove (2.10). This difficulty appears to be purely an algebraic one, and no attempt is made here to overcome it.

\section{Non-existence}

The proof of the non-existence part of (2.10) depends on the Fredholm alternative property of any linear operation $E$ on a Hilbert space: $\dagger$

A given linear, non-homogeneous equation,

$$
E x=b,
$$

has a solution only if every non-trivial solution to the homogeneous adjoint equation,

satisfies

$$
E^{*} y=0
$$

where

$$
\langle b, y\rangle=0 \text {, }
$$

$$
\langle b, y\rangle=\sum_{n=1}^{\infty} b_{n} y_{n}
$$

If $E$ is a real matrix, then $E^{*}$ is simply the transpose of that matrix.

Let us impose restrictions on the range of the parameters again, which we will remove after completing the proof. We consider as a prototype the asymmetric case in which

$$
[k]=1, \quad[k w]=0 \text { and } \mu_{1}=0 .
$$

The transpose $E^{*}$ of the matrix $E$ of (2.7) then is given by

$$
E^{*}=\left(\begin{array}{cccc}
B_{12} & -A_{22} & B_{32} & \cdots \\
B_{13} & B_{23} & -A_{33} & \cdots \\
B_{14} & B_{24} & B_{34} & \cdots \\
\vdots & \vdots & \vdots &
\end{array}\right)
$$

where the $A_{m n}$ and $B_{m n}$ are given by (A 2) and (A 3). Let the solution (if any) to (A $13 b$ ) be denoted by $\left\{y_{n}\right\}_{n=1}^{\infty}$. Then the homogeneous adjoint equation corresponding to (2.7), when every term involving $y_{1}$ is transferred to the right-hand side, takes the form

$$
\left(\begin{array}{cccc}
-A_{22} & B_{32} & B_{42} & \cdots \\
B_{23} & -A_{33} & B_{43} & \cdots \\
B_{24} & B_{34} & -A_{44} & \cdots \\
\vdots & \vdots & \vdots &
\end{array}\right)\left(\begin{array}{c}
y_{2} \\
y_{3} \\
y_{4} \\
\vdots
\end{array}\right)=-y_{1}\left(\begin{array}{c}
B_{12} \\
B_{13} \\
B_{14} \\
\vdots
\end{array}\right)
$$

But the matrix in (A 15) is exactly the kind that was just shown to have a wellbehaved inverse. Therefore, the homogeneous adjoint equation has a non-trivial 
solution. From (A 3), $\left\{B_{1 n}\right\}_{n=2}^{\infty} \in l^{2}$, so $\left\{y_{n}\right\}_{n=1}^{\infty} \in l^{2}$ as well. Equation (A 13c) can be written as

$$
f_{1} y_{1}=-\sum_{n=2}^{\infty} f_{n} y_{n}
$$

where $\left\{f_{n}\right\}$ is defined by $(2.7 a)$. Observe that

$$
\sum_{n=1}^{\infty}\left|\frac{f_{n}}{n^{\frac{1}{2}}}\right|<\infty ; \text { hence } \sum_{n=2}^{\infty} f_{n} y_{n}
$$

is ordinarily too small to satisfy (A 16). It is possible that (A 16) can be satisfied if $f_{1}=0$. A symmetric contraction yields $f_{1}=0$, but in that case (2.7) is degenerate. For an asymmetric contraction, $f_{1}=0$ only for a discrete set of combinations of $\left(k, \mu_{1}, \mu_{2}\right)$. (For example, in the asymmetric prototype, $\mu_{1}=0,[k]=1$, there exist at most two non-trivial values of $\mu_{2}$ such that $f_{1}=0$ in (2.7).) With the possible exception of this discrete set of combinations of $\left(k, \mu_{1}, \mu_{2}\right)$ (A 16) cannot be satisfied, so that (2.7) has no solution.

The restrictions placed on the parameters are easily removed. For some other combination of $[k]$ and $[k w]$, the right-hand side of (A 15) would be composed of a linear combination of terms like $-y_{n}\left\{B_{n j}\right\}_{j=[k w]}^{\infty}$, with $[k w]<n \leqslant[k]$. The rest of the proof has similar changes.

The restriction to one-sided contractions can be removed to the extent that it was removed in the proof of the first part, since this proof depends on the results of that part. Thus, for $k<1,(2.10)$ holds for any contraction. For arbitrary $k$, (2.10) is established for one-sided contractions and for symmetric contractions.

It is worth noting explicitly that, as $k$ increases, the value of $w$ below which no solution exists approaches unity, so that Long's model yields no solution for any particular contraction if $k$ is large enough. This completes the proof of $(2.10)$.

\section{Appendix B. The inviscid flow past rectangular obstacles}

The solution to the boundary-value problem corresponding to (2.3) for rectangular obstacles (see figure 3 ) can be determined by separation of variables, as was the flow field in $\$ 2$. Thus,

$$
\begin{gathered}
\delta=\sum_{n=N+1}^{\infty} \frac{A_{n}}{n^{\frac{1}{2}}} \exp \left[\left(n^{2}-k^{2}\right)^{\frac{1}{2}} x\right] \sin n y \quad(x<0), \\
\delta=\sum_{n=1}^{R}\left\{\frac{D_{n} \sin \left(k^{2}-(n / w)^{2}\right)^{\frac{1}{2}} x}{n^{\frac{1}{2}} \sin \left(k^{2}-(n / w)^{2}\right)^{\frac{1}{2}} L}-\frac{E_{n} \sin \left(k^{2}-(n / w)^{2}\right)^{\frac{1}{2}}(x-L)}{n^{\frac{1}{2}} \sin \left(k^{2}-(n / w)^{2}\right)^{\frac{1}{2}} L}\right\} \sin \frac{n}{w}(y-h) \\
+\sum_{n=R+1}^{\infty}\left\{\frac{D_{n} \sinh \left((n / w)^{2}-k^{2}\right)^{\frac{1}{2}} x}{n^{\frac{1}{2}} \sinh \left((n / w)^{2}-k^{2}\right)^{\frac{1}{2}} L}-\frac{E_{n} \sinh \left((n / w)^{2}-k^{2}\right)^{\frac{1}{2}}(x-L)}{n^{\frac{1}{2}} \sinh \left((n / w)^{2}-k^{2}\right)^{\frac{1}{2}} L}\right\} \sin \frac{n}{w}(y-h) \\
+\gamma \sin k(y-\tilde{y}) \quad(0<x<L), \\
\delta=\sum_{n=1}^{N}\left\{\frac{B_{n}}{\left.n^{\frac{1}{2}} \cos \left(k^{2}-n^{2}\right)^{\frac{1}{2}}(x-L)-\frac{C_{n}}{n^{\frac{1}{2}}} \sin \left(k^{2}-n^{2}\right)^{\frac{1}{2}}(x-L)\right\} \sin n y}\right. \\
\quad+\sum_{n=N+1}^{\infty} \frac{B_{n}}{n^{\frac{1}{2}}} \exp \left[-\left(n^{2}-k^{2}\right)^{\frac{1}{2}}(x-L)\right] \sin n y \quad(x>L), \quad \text { (B 1) }
\end{gathered}
$$


where $w=(\pi-h) / \pi, N=[k], R=[k w]$, and $L$ is restricted by

$$
\sin \left(k^{2}-(n / w)^{2}\right)^{\frac{1}{2}} L \neq 0 \text { for all } n<R .
$$

Upon elimination of $\left\{B_{n}\right\}_{1}^{[k]},\left\{D_{n}\right\}_{1}^{\infty}$ and $\left\{E_{n}\right\}_{1}^{\infty}$ one is left with an infinite set of linear algebraic equations of the following form: $\dagger$

in which

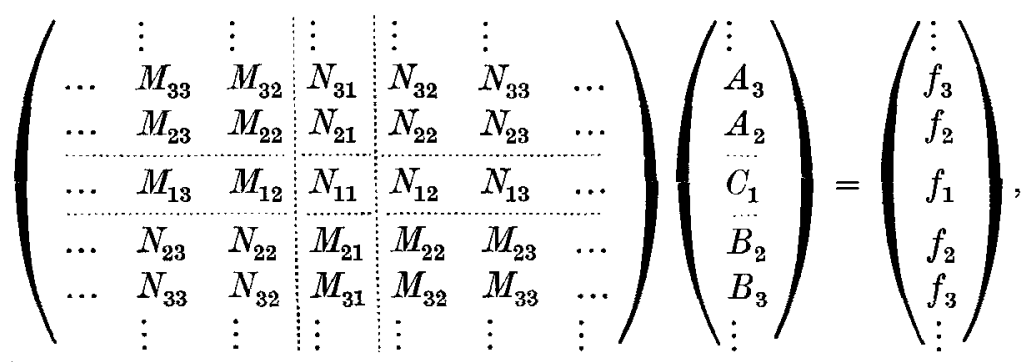

$$
\begin{aligned}
& M_{m n}= \frac{\pi}{2} \delta_{m n}+\frac{2}{\pi} w(m n)^{\frac{1}{2}}\left|1-\left(\frac{n}{k}\right)^{2}\right|^{\frac{1}{2}} \sin (n h) \sin (m h) \\
& \times \sum_{r=1}^{\infty} r^{2} d(m, r) d(n, r) s(r) \operatorname{coth}\left(s^{-1}(r) L\right) \\
& N_{m n}=\frac{2}{\pi} w(m n)^{\frac{1}{2}}\left|1-\left(\frac{k}{n}\right)^{2}\right|^{\frac{1}{2}} \sin (n h) \sin (m h) \\
& \times \sum_{r=1}^{\infty} r^{2} d(m, r) d(n, r) s(r) \operatorname{cosech}\left(s^{-1}(r) L\right),
\end{aligned}
$$

$d, s$ are defined by (2.7), and $\delta_{m n}$ is the Kronecker delta. The $\left\{f_{n}\right\}$ are identical with those of (2.7) for $\mu_{1}=h, \mu_{2}=\pi$. Significantly, they are independent of $L$. Following the pattern of $\S 2$, one can show that this matrix has dominant diagonal elements. In fact, $M_{m n}$ shows the same dependence on $(m, n)$ as did the matrix elements in $\$ 2$. For large $m$ or $n$,

$$
\begin{aligned}
M_{m n} & \doteq \frac{2}{\pi} \sin n h \sin m h \frac{(n m)^{\frac{1}{2}}}{m^{2}-n^{2}} \ln \frac{n}{m} \quad \text { if } \quad m \neq n, \\
M_{n n} & \doteq \frac{1}{2} \pi+2 \pi w \alpha(n),
\end{aligned}
$$

where

$$
0<\alpha(n)<4 / \pi^{2} \text {. }
$$

Similarly, one establishes the following bounds on $N_{m n}$ for large $m$ or $n$ :

$$
\begin{aligned}
&\left|N_{m n}\right|<2 w \tanh ^{-1}\left(\exp \left[-\frac{L}{w}\left\{([k w]+1)^{2}-(k w)^{2}\right\}^{\frac{1}{2}}\right]\right) \frac{(n m)^{\frac{1}{2}}}{\left|m^{2}-n^{2}\right| L} \text { if } m \neq n \\
&\left|N_{n n}\right|<\frac{32}{9 \pi} \tanh ^{-1}\left(\exp \left[-\frac{L}{w}\left\{([k w]+1)^{2}-(k w)^{2}\right\}^{\frac{1}{2}}\right]\right) \frac{1}{n^{2} L} .
\end{aligned}
$$

The magnitude of $N_{m n}$ for $m, n<k$ is quite important. If $[k]=1$, either [kw] $=1$

$\dagger$ We shall consider $[k]=1$ as a prototype in all of the subsequent development. 
or $[k w]=0$. If $[k w]=0$, one can show that the first term in the infinite sum in (B 2c) dominates, so that

$$
N_{11} \doteq \frac{2 \pi w}{(1+w)^{2}}\left(\frac{\sin h}{h}\right)^{2}\left(\frac{k^{2}-1}{1-(k w)^{2}}\right)^{\frac{1}{2}} / \sinh \left(\frac{L}{w}\left\{1-(k w)^{2}\right\}^{\frac{1}{2}}\right) .
$$

If $[k w]=1$, the first term involves trigonometric, rather than hyperbolic, functions, so that

$$
N_{11} \doteq\left\{-\frac{2 \pi w}{(1+w)^{2}}\left(\frac{\sin h}{h}\right)^{2}\left(\frac{k^{2}-1}{(k w)^{2}-1}\right)^{\frac{1}{2}} / \sin \left(\frac{L}{w}\left((k w)^{2}-1\right)^{\frac{1}{2}}\right)\right\}+O\left(L^{-1}\right) .
$$

(B 4) displays the importance of both the criterion (2.5) and the obstacle length $L$ in determining the behaviour of the matrix in (B 2). The matrix becomes more nearly singular as $L$ increases (and $N_{11}$ vanishes) only if [k] $>[\mathrm{kw}]$.

Following appendix A, one would like to show that the matrix in (B 2) has an inverse which is defined by a Neumann series, similar to (A 6). From that result one could then deduce that $C_{1} \doteq f_{1} / N_{11}$.

When $[k]>[k w]$ and $L$ is large, however, so that the matrix is nearly singular, it is not obvious that any such representation exists. Hence, we turn to the following suggestive line of reasoning, which does not constitute a proof.

Consider first the truncated set of equations, obtained from those in (B 2) by setting $N_{m n}=0$ for $n>1$ :

$$
\left(\begin{array}{llll} 
& \vdots & \vdots & \vdots \\
\ldots & M_{33} & M_{32} & N_{31} \\
\ldots & M_{23} & M_{22} & N_{21} \\
\ldots & M_{13} & M_{12} & N_{11}
\end{array}\right)\left(\begin{array}{l}
\vdots \\
A_{3} \\
A_{2} \\
C_{1}
\end{array}\right)=\left(\begin{array}{l}
\vdots \\
f_{3} \\
f_{2} \\
f_{1}
\end{array}\right)
$$

This set of equations is equivalent to the following set:

$$
\left(\begin{array}{llll} 
& \vdots & \vdots & \vdots \\
\ldots & M_{33} & M_{32} & \tilde{M}_{31} \\
\ldots & M_{23} & M_{22} & \tilde{M}_{31} \\
\ldots & M_{13} & M_{12} & \frac{1}{2} \pi
\end{array}\right)\left(\begin{array}{l}
\vdots \\
A_{3} \\
A_{2} \\
\tilde{C}_{1}
\end{array}\right)=\left(\begin{array}{l}
\vdots \\
f_{3} \\
f_{2} \\
f_{1}
\end{array}\right),
$$

in which $\widetilde{C}_{1}=(2 / \pi) N_{11} C_{1}$, and $\widetilde{M}_{n 1}=\frac{1}{2} \pi N_{n 1} / N_{11}$.

But (B 5) is almost exactly (2.7) with $[k]=[k w]$. Certainly a Neumann series exists for the inverse of this matrix, so that

Further,

$$
\tilde{C}_{1} \doteq(2 / \pi) f_{1} \quad \text { or } \quad C_{1} \doteq f_{1} / N_{11}
$$

(Note that the result, (B 6a), does not depend on the value of $[k w]$.)

Consider next a different truncation of (B 2), obtained by setting $N_{m n}=0$ for $n, m>1$ and $M_{m n}=0$ for $m=1, n>1$ :

$$
\left(\begin{array}{llll}
N_{11} & N_{12} & N_{13} & \cdots \\
M_{21} & M_{22} & M_{23} & \cdots \\
M_{31} & M_{32} & M_{33} & \cdots \\
\vdots & \vdots & \vdots &
\end{array}\right)\left(\begin{array}{l}
C_{1} \\
B_{2} \\
B_{3} \\
\vdots
\end{array}\right)=\left(\begin{array}{l}
f_{1} \\
f_{2} \\
f_{3} \\
\vdots
\end{array}\right)
$$


These equations, in turn, can be rewritten as

$$
\left(\begin{array}{llll}
\frac{1}{2} \pi & \tilde{M}_{12} & \tilde{M}_{13} & \cdots \\
M_{21} & M_{22} & M_{23} & \cdots \\
M_{31} & M_{32} & M_{33} & \cdots \\
\vdots & \vdots & \vdots &
\end{array}\right)\left(\begin{array}{l}
C_{1} \\
B_{2} \\
B_{3} \\
\vdots
\end{array}\right)=\left(\begin{array}{l}
\tilde{f}_{1} \\
f_{2} \\
f_{3} \\
\vdots
\end{array}\right)
$$

where $\tilde{f_{1}}=\frac{1}{2} \pi f_{1} / N_{11}$ and $\tilde{M}_{1 n}=\frac{1}{2} \pi N_{1 n} / N_{11}$.

Again, this set is almost exactly (2.7) with $[k]=[k w]$, except that $\tilde{f}_{1}$ might be much larger here (if $N_{11}$ is very small). Again, a Neumann series exists, and

If

$$
C_{1} \doteq(2 / \pi) \tilde{f}_{1} \quad \text { or } \quad C_{1} \doteq f_{1} / N_{11}
$$

if

$$
\begin{array}{ll}
N_{11}=O(1), & B_{n} \doteq f_{n} / M_{n n} ; \\
N_{11}=o\left(L^{-1}\right), & B_{n}=O\left(N_{11}^{-1}\right) .
\end{array}
$$

Thus, one obtains the result (B 6) for $N_{n n}=0$ if $m, n>1$ in (B 2). But, from (B 3) to (B 4), one notes that the actual values of these $N_{m n}$ are small:

$$
\begin{aligned}
& \frac{N_{m n}}{N_{11}}=O\left(\frac{1}{L\left(m^{2}-n^{2}\right)}\right) \text { if } m \neq n \\
& \frac{N_{n n}}{N_{11}}=O\left(\frac{1}{L n^{2}}\right) \quad \text { if } \quad n>1 .
\end{aligned}
$$

Certainly for $L$ large enough, then, one could obtain yet another Neumann series for the matrix $E$ in (B 2):

$$
E^{-1}=E_{1}^{-1}\left(I-E_{2} E_{1}^{-1}+E_{2} E_{1}^{-1} E_{2} E_{1}^{-1} \ldots\right),
$$

where $E_{1}$ comprises all of the elements of both truncated matrices considered above, and $E_{2}$ comprises all of the elements of the matrix in (B 2) which were ignored in both truncations.

Algebraic complications preclude one's obtaining a close enough bound on $E_{2}$ to prove this result by the methods used above. Proving it would prove not only (B 7); it would prove as well Drazin \& Moore's claim that solutions always exist for rectangular obstacles, and confirm the conjecture mentioned above that, for a barrier which reflects almost all the energy from its wave-generator, the wave-pattern downstream is quite intense.

It follows that (B 6) is a first approximation to the full solution of (B 2). Combining this result with (B 4) and dropping the restriction $[k]=1$, one obtains the desired result:

$$
\begin{array}{ll}
\text { if } \quad[k]=k[w], & C_{n}=O(1) \quad \text { as } \quad L \rightarrow \infty \\
\text { if } \quad[k]>[k w], & \text { ord }\left(C_{n}\right)>L \quad \text { as } \quad L \rightarrow \infty .
\end{array}
$$

This establishes (3.1).

\section{REFERENCES}

BALL, F. K. 1959 Long waves, lee waves and gravity waves. Quart. J. Roy. Met. Soc. 85, 24-30.

Benjamin, T. B. 1970 Upstream influence. J. Fluid Mech. 40, 49-80. 
DAvis, R.E. 1969 The two-dimensional flow of a stratified fluid over an obstacle. J. Fluid Mech. 36, 127-143.

Debler, W. R. 1959 Stratified flow into a line sink. J. Eng. Mech. Div., Proc. ASCE, $85,51-65$.

DraziN, P. G. \& Moore, D. W. 1967 Steady two-dimensional flow of fluid of variable density over an obstacle. J. Fluid Mech. 28, 353-370.

DubReIL-JACotin, M. L. 1935 Complément à une note antérieure sur les ondes de type permanent dans les liquides hétérogènes. Atti Accad. Naz. Lincei Rc. (Cl. Sci. Fis. Mat. Nat.) (6), 21, 344-346.

Friedman, B. 1956 Principles and T'echniques of Applied Mathematics. New York: Wiley.

Grimshaw, R. 1968 A note on the steady two-dimensional flow of a stratified fluid over an obstacle. J. Fluid Mech. 33, 293-301.

Hardy, G. H., LitTlewood, J. E. \& Polya, G. 1952 Inequalities. Cambridge University Press.

KAO, T. W. 1965 The phenomenon of blocking in stratified flows. J. Geophys. Res, 70, $815-822$.

Long, R. R. $1953 a$ Some aspects of the flow of stratified fluids, I. Tellus, 5, 42-58.

LoNg, R. R. $1953 b$ Steady motion around a symmetrical obstacle moving along the axis of a rotating liquid. $J$. Meteorology, 10, 197-203.

LoNG, R. R. 1955 Some aspects of the flow of stratified fluids. III. Continuous density gradients. Tellus, 7, 341-357.

TRustrum, K. 1964 Rotating and stratified fluid flow. J. Fluid Mech. 19, 4I5-432.

YIH, C. C. 1965 Dynamics of Non-homogeneous Fluids. New York: Macmillan. 